\title{
Optimization of suspensions filtration with compressible cake
}

\author{
Dagmar Janacova ${ }^{1, a}$, Pavel Mokrejs ${ }^{2}$, Karel Kolomaznik ${ }^{1}$, Vladimir Vasek $^{1}$, Rudolf Drga ${ }^{1}$, Ondrej Líska ${ }^{3}$ and Jiri Krenek \\ ${ }^{1}$ Tomas Bata University in Zlin, Faculty of Applied Informatics, nám. T.G.Masaryka 5555, 76001 Zlín. Czech Republic \\ ${ }^{2}$ Tomas Bata University in Zlin, Faculty of Technology, nám. T.G.Masaryka 5555, 76001 Zlín. Czech Republic \\ ${ }^{3}$ University of Košice, Faculty of Mechanical Engineering, Letná 9, 04200 Košice, Slovak Republic
}

\begin{abstract}
In this paper there is described filtering process for separating reaction mixture after enzymatic hydrolysis to process the chromium tanning waste. Filtration of this mixture is very complicated because it is case of mixture filtration with compressible cake. Successful process strongly depends on mathematical describing of filtration, calculating optimal values of pressure difference, specific resistant of filtration cake and temperature maintenance which is connected with viscosity change. The mathematic model of filtration with compressible cake we verified in laboratory conditions on special filtration device developed on our department.
\end{abstract}

\section{Introduction}

Filtration belongs to the most common cases practically making use of phenomena taking place during the flow of liquid through a layer of granular material. Its industrial application is similar to laboratory filtration performed on filter paper in a filter funnel. The objective is to separate solid particles from the liquid in which they are dispersed. This separation is achieved during filtration by forcing the liquid containing solid particles through a porous barrier. Solid particles get caught in pores of the barrier and gradually build up a layer on it. Liquid passes through the particle layer and porous barrier and leaves, gradually freed of its solid phase.

\section{Filtration}

A suspension of solid particles in liquid is brought to the filter, and filtrate and filter cake are drawn off [5]. Let us designate: $m_{F}$ - mass of filtrate, $m_{S}$ - mass of suspension, $m_{K}$ - mass of filter cake, $w_{F}$ - mass fraction of solid phase concentration in filtrate, $w_{S}$ - mass fraction of solid phase in suspension, $w_{K}$ - mass fraction of solid phase in cake. Balance relationships are balances of whole flows as well as balances of both phases. If the filter operates efficiently, value $w_{F}$ is very small and negligible in relation to the others and the quantity of filtrate can then be determined from the quantity of suspension and concentrations of solid particles in suspension and filter cake:

$$
m_{F}=m_{S}\left(1-\frac{w_{S}}{w_{K}}\right)
$$

$$
p_{1}-p_{2}=\rho\left(\frac{\lambda h A v_{F}^{2}}{8 \varepsilon^{3}}+g h\right)
$$

where: $p_{1}$ - pressure before cake, $p_{2}$ - pressure behind cake, $\rho$ - density, $v_{F}$ - velocity, $\lambda$ - friction coefficient, $\varepsilon$-porosity of layer, $g$ - gravitational constant, $h$ - thickness of cake, $A$ - surface density of articles.

Term $g h$ usually has no significance in filtration as the hydrostatic pressure produced by height of suspension level is generally negligible when compared with a pressure difference produced by industrial means [1], [4]. Provided the particles are sufficiently firm and do not undergo deformation, porosity remains constant throughout filtration and the filter cake is regarded as incompressible. Constant $C$ and cake properties, i.e. porosity and surface density, then combine into a single constant:

$$
\alpha=C A^{2} / \varepsilon^{3}
$$

after simplification:

$$
p_{1}-p_{2}=\alpha h \mu v_{F}
$$

Practical account is taken of the difference between $p_{I}$ and $p_{3}$. When passing through this screen, filtrate changes its pressure from $p_{2}$ to $p_{3}$ because the screen offers resistance to its flow

$$
p_{2}-p_{3}=R v_{F}
$$

Combining equations for filter cake and screen, we obtain the filtration equation:

\footnotetext{
${ }^{\mathrm{a}}$ Corresponding author: janacova@fai.utb.cz
} 


$$
p_{1}-p_{3}=(\alpha h \mu+R) v_{F}
$$

The flow of filtrate through cake is described as a flow of liquid through a layer of granular material [7]. This equation is used more practically in the form containing volume of

filtrate:

$$
\frac{d V_{F}}{d \tau}=\frac{\left(p_{1}-p_{3}\right) S^{2}}{\beta \mu\left(V+V_{E K V}\right)}
$$

where

$$
\beta=\alpha \tau \frac{V_{K}}{V_{F}}
$$

and $V_{E K V}$ is the resistance of filter screen expressed by means of equivalent thickness of filter cake. Integration of (7) gives:

$$
\begin{aligned}
& V^{2}+V_{E K V} V=2 K \tau \\
& \text { where } \quad K=\frac{\left(p_{1}-p_{2}\right) S^{2}}{\beta \mu}
\end{aligned}
$$

With a constant rate of filtrate flow, the solution of (7) is:

$$
V^{2}+V_{E K V} V=K \tau
$$

Accordingly, the deformation of particles has to be taken into account as this affects particle porosity. In that case specific resistance of the filter cake is not constant. On passage through the cake, filtrate pressure drops from value $p_{1}$ to $p_{2}$. Deformation of the cake is caused by a resistive force when the stream flows past particles in a layer placed on a firm screen. The intensity of resistance $F^{\prime}{ }_{R}$ equals the difference in pressures $\left(p_{1}-p_{2}\right)$ multiplied by flow cross-section, $S$ - area of filter:

$$
F_{R=}^{\prime}\left(p_{1}-p_{2}\right) S
$$

As pressure $\mathrm{p}$ decreases when going from the interface with suspension to interface with screen, resistive force in the same direction increases and this is the force causing deformation of particles. This deformation then affects value of $\alpha$ and/or $\beta$. A certain value is found only across a differential section of the cake between $h^{\prime}$ and $h^{\prime}+d h^{\prime}$ described by equation:

$$
-d p=\alpha \eta v_{F} d h
$$

Integration across the whole thickness of cake and substitutions $d p^{\prime}=-d p$ and $d h=-d h^{\prime}$ give:

$$
\left.\int_{0}^{p-p_{2}} \frac{d p}{\alpha}=-\int_{0}^{-h}-\mu v_{F} d h \Rightarrow p_{1}-p_{2}=\right\rangle \alpha\left\langle\eta v_{F} h\right.
$$

where $\langle\alpha\rangle$ is the mean value of $\alpha$.
The mean value of $\alpha(\beta)$ is determined from the dependence on $p^{\prime}$. It is found by filtration tests in an apparatus where value of $p^{\prime}$ can be altered. This dependence is usually expressed in the form as follows:

$$
\alpha=\alpha_{0}\left[1+\beta(p)^{\gamma}\right]
$$

Equation for rate of filtration:

$$
v_{F}=\frac{p_{1}-p_{2}}{\langle\alpha\rangle \mu h+R}=\frac{\Delta p \cdot S^{2}}{\langle\beta\rangle \mu\left(V+V_{E K V}\right)}
$$

$$
p_{s}=\frac{1}{\tau_{1}-\tau_{2}} \int_{\tau_{2}}^{\tau_{2}} f(\tau) d \tau
$$

Calculated mean values are presented in the following table [2]. Parameter $\beta$ characterizes filter cake resistance. If this does not change when pressure differences during filtration change, the cake in question is incompressible; on the contrary, it is compressible if resistance changes.

We adapted the filtration equation for a constant pressure difference $[1,6]$ :

$$
\frac{d V_{i}}{d t}=\frac{\Delta p \cdot S_{f}^{2}}{\mu \beta \cdot V_{i}}
$$

Assuming that $\Delta p=p_{s i}$, and that $\beta_{i}, \mu, \Delta p$ and $\mathrm{S}_{f}^{2}$ are constant within individual intervals and can be substituted by $K_{i}$, Constant $K$ enabled us to determine:

$$
\beta_{i}=\frac{\Delta p \cdot S_{f}^{2}}{\mu \cdot K_{i}}
$$

The dependence was approximated by a $d x$ potential

$$
\beta=1,76 \cdot 10^{14} \cdot e^{7,7275 \cdot 10^{5} \cdot p_{s}}
$$

For industrial application, the best advantage is gained at greatest rate (maximum filtrate in time). Therefore, the given equation was differentiated with respect to pressure difference:

$$
\Delta p: \quad \frac{d v}{d \Delta p}=\frac{S_{f}^{2}}{\mu \cdot V_{F}} \cdot \frac{B \cdot e^{C . \Delta p}-\Delta p \cdot B \cdot C \cdot e^{C . \Delta p}}{\left(B \cdot e^{C . \Delta p}\right)^{2}}
$$

\section{Experimental part}

In laboratory conditions we verified recycling technology of leather shavings treatment. On the figure 1 is shown easier technology scheme of enzymatic hydrolysis [1].

The laboratory filtration model was designed to meet following requirements [3]:

- constant temperature of $30-80{ }^{\circ} \mathrm{C}$ in filtering space,

- filter underpressure recording (approx. 0,6 kPa),

- filtrate volume 51 


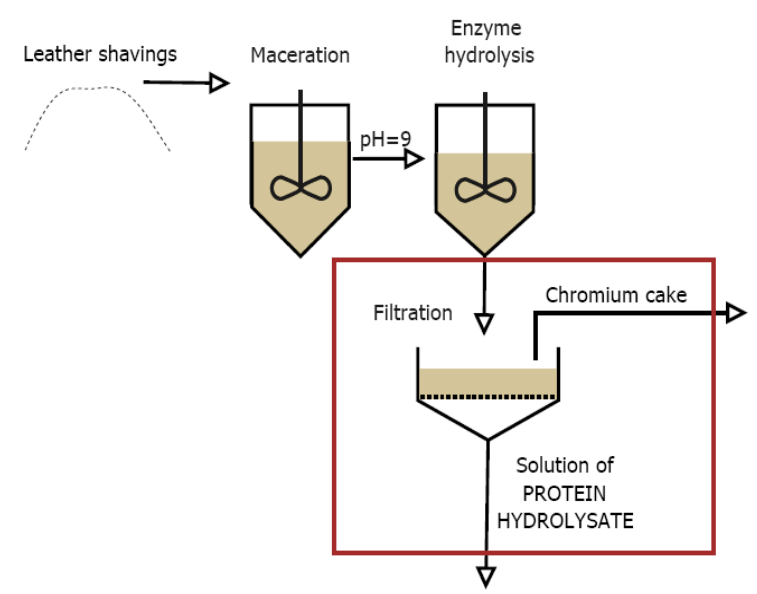

Figure 1. Process of enzymatic hydrolysis - scheme.

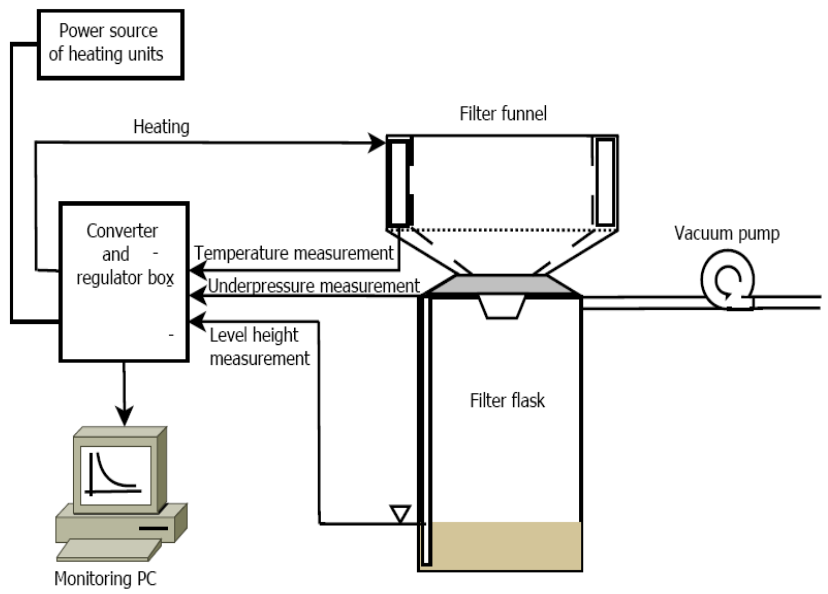

Figure 2. Laboratory arrangement of filtration.

The model consists of parts as follows:

- temperature-controlled heated filter funnel,

- filtration flask,

- underpressure measurement,

- level height measurement,

- unified signal conversion,

- data storage.

The filter funnel of circular shape consists of two main parts, the funnel proper and filter screen. Both parts were made of dural, which is light and conducts heat well [3], [4].

The basic dimensions of the funnel are inside diameter of $16,5 \mathrm{~cm}$ and funnel edge of $8 \mathrm{~cm}$ height. It can be filled at a time with 1.7 litres of the filtered suspension. The funnel wall is provided with 4 holes drilled symmetrically from the bottom side for holding heating elements. Another two holes are asymmetrically drilled there for resistance thermometers. One of these is located in the close vicinity of a heating element, the second is precisely between these elements. The resistance thermometers are connected in series, which in the chosen spacing permits better determination of the funnel average temperature.

Complete sealing during filtration induced by underpressure under the funnel is provided by two rubber packing. The first of annulus shape is between the funnel proper and filter screen. The second, suitably shaped packing is mounted between the filter funnel and mouth of filtration flask [3].

Heating was provided by 4 heating elements fed by $24 \mathrm{~V}$ a.c., each of $60 \mathrm{~W}$ power. The heating bodies were divided into two parallel sections, each containing two bodies connected in series. The heating bodies were powered through an external transformer.

Filtration was performed while the funnel was heated to $50^{\circ} \mathrm{C}$. Properties of the suspension were as follows:

density $\rho=2500 \mathrm{kgm}^{-3}$, dynamic viscosity $\mu=0,001$ Pas. Primary filtration proceeded for the first 100 seconds without external underpressure, merely under own hydrostatic pressure. After 100 seconds, underpressure was produced by the vacuum pump. At the time when an underpressure decrease characterizing stoppage of filtration appeared, the value of underpressure was increased. This was repeated until the cake cracked and filtration stopped. An analogous procedure was employed in secondary filtration.

Primary filtration is the term applied to filtration in progress during the first stage of enzymatic dechromation, secondary filtration applied to that during the second stage. The pressure difference means value of produced underpressure or value at the beginning of hydrostatic pressure and both terms are interchangeable.

The course of pressure differences during primary filtration of first-stage suspension is depicted in Figure 3.

Four stages are obvious in primary filtration. The first (0-100 s) without underpressure, second (100-460 s) with underpressure exerted for the first time. Further underpressure increases occurred in the third (460-920 s) and fourth (920-2490 s) stages. A drop in underpressure follows stage four - the already mentioned filter cake rupture.

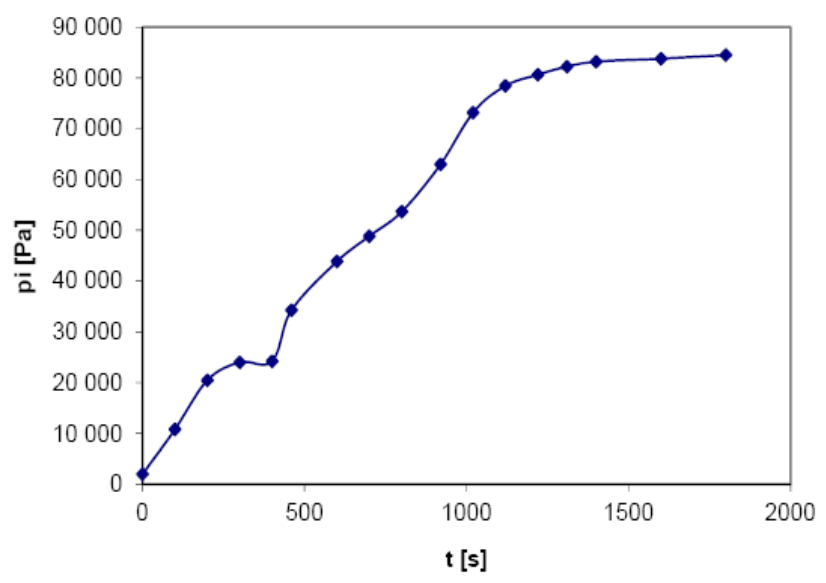

Figure 3. Course of underpressure during primary filtration

This signifies that the specific resistance of filter is independent of pressure difference $\Delta p=12940 \mathrm{~Pa}$ (equation 21). 


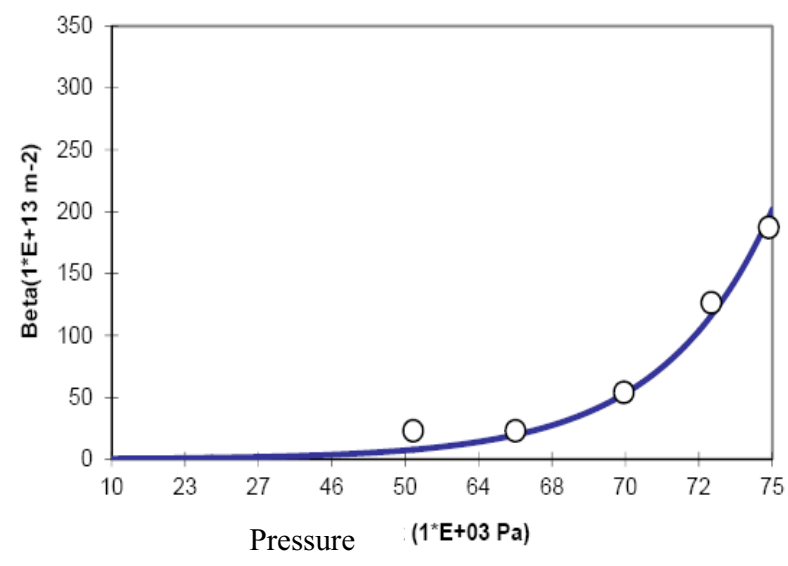

Figure 4. Dependence of parameter $\beta$ on difference approximation by exponential curve (equation 20)

The procedure in secondary filtration of hydrolysate was the same as in primary filtration. Calculated values are presented in the table as follows.

Table 1. Calculated values for primary filtration.

\begin{tabular}{|c|c|c|c|c|}
\hline $\begin{array}{c}\text { Interval } \\
\text { No. }\end{array}$ & $\begin{array}{c}\tau_{\boldsymbol{I}} \\
{[\mathrm{s}]}\end{array}$ & $\begin{array}{c}\tau_{2} \\
{[\mathrm{~s}]}\end{array}$ & $\begin{array}{c}\boldsymbol{P}_{\boldsymbol{s}} \\
{[\mathrm{Pa}]}\end{array}$ & $\begin{array}{c}\boldsymbol{\beta} \\
{\left[\mathrm{m}^{-2}\right]}\end{array}$ \\
\hline I & 0 & 50 & 1962 & $4,3 \mathrm{E}+12$ \\
\hline II & 50 & 80 & 10802 & $2,4 \mathrm{E}+12$ \\
\hline III & 80 & 300 & 26813 & $5,6 \mathrm{E}+12$ \\
\hline
\end{tabular}

Filtration of a similar volume of suspension proceeded much faster (about 22 times). Values of the cake specific resistance varied merely within the same order; the cake, therefore, was further considered to be incompressible. The general expression for calculating optimal values of pressure difference we used for control of primary filtration of suspension. The process having started, filtration runs without external cause only due to its own hydrostatic pressure. When the defined sampling period has elapsed, constant $\mathrm{K}$ is and the value of inductive force is increased by a difference defined in advance. With this first incrementation, the source of force changes from pressure to underpressure. Constant $\mathrm{K}$ is again calculated after this period, namely by recording changes in height of level. The preceding value of $\mathrm{K}$ is compared with the present. If it fails to comply with the specified condition, underpressure is increased again, and $\mathrm{K}$ is newly calculated after the time difference has elapsed. The cycle is terminated upon condition being met. A further condition follows, comparing actual underpressure with the value of optimal underpressure found in identifying filtration. Control is finished upon reaching accordance within a certain tolerance. If values of underpressures differ, the value found during process modelling is set up and control is finished. Parameter $\mathrm{x}$ of the first condition is chosen a priori and has an influence on underpressure being possibly exceeded. If $\mathrm{x}$ were chosen with a value approaching 1 from below, filtration would last too long.

\section{Conclusion}

In this contribution we have successfully solved the problem of separation of heterogeneous mixtures after enzymatic hydrolysis in the case of production of gelatine protein. By using controlled filtration during her constant speed, we have increased production of gelatine protein. We determined optimal underpressure and parameter $\beta$ for filtration process with compressible cake. The filtration process we verified on our developed filtration equipment in laboratory conditions. For another suspensions with compressible cake we can determine optimal parameters: underpressure, specific resistance $\beta$ and time of filtration also.

\section{Acknowledgement}

This work was supported by the Ministry of Education, Youth and Sports of the Czech Republic within the National Sustainability Programme project No. LO1303 (MSMT-7778/2014) and also by the European Regional Development Fund under the project CEBIA-Tech No. CZ.1.05/2.1.00/03.0089

\section{References}

1. K. Kolomazník, Recovery and recycling of chromium and protein from liquid and solid wastes from leather industry, ERB 3512 PL 940974 (COP 974), (1996)

2. D. Janacova, K. Kolomaznik, V. Vasek, Optimization of liquids consumption at washing. 6th International Carpathian Control Conference. Miskolc-Lilafuered, Hungary, ISBN 963-661-644-2. (2005)

3. V. Vasek, K. Kolomaznik, D. Janacova, Optimization and Automatic Control of Chromium Recycling Technology, WSEAS Transactions on Systems, 5 (11), ISSN 2651-2654. (2006)

4. J. Dolinay, P. Dostalek, V. Vasek, New Embedded Control System for Enzymatic Hydrolysis, Proceedings of the 8th WSEAS International Conference on Applied Informatics and Communications, Rhodes, Greece, p. 174, ISBN: 978-960-6766-94-7. (2008)

5. L. Vasek, V. Dolinay Simulation model of heat distribution and consumption in municipal heating network, International Journal of Mathematical Models and Methods in Applied Sciences, Vol. 4 (4), p. 240-248. (2010)

6. J. Pitel, J. Borzikova, Mizak, J. Biomass combustion process control using artificial intelligence techniques, Proceedings of $X X X V$. Seminar ASR'2010 Instruments and Control. (2010)

7. K. Kyas, J. Cerný, M. Stanek, M. Manas, D. Manas, V. Senkerik, A. Skrobak, Measuring of temperature and pressure in injection mold, International Journal of Mathematics and Computers in Simulation., Vol. 6 (6), p. 600-607. (2012) 